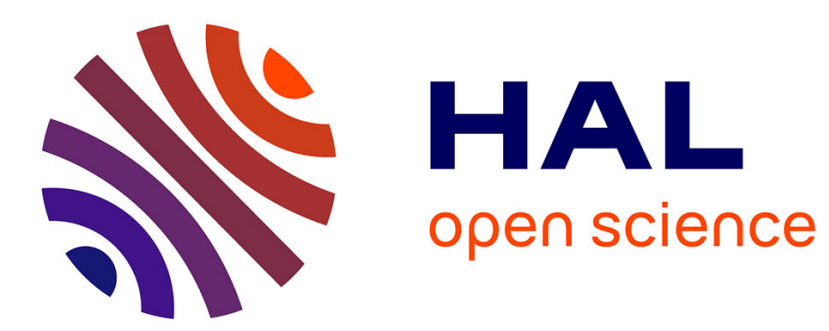

\title{
A Framework for Mathematical Analysis of Collaborative SCM in ColPMan Game
}

\author{
Tatsuki Furukawa, Tomomi Nonaka, Hajime Mizuyama
}

\section{To cite this version:}

Tatsuki Furukawa, Tomomi Nonaka, Hajime Mizuyama. A Framework for Mathematical Analysis of Collaborative SCM in ColPMan Game. IFIP International Conference on Advances in Production Management Systems (APMS), Sep 2017, Hamburg, Germany. pp.311-319, 10.1007/978-3-319-669267_36.hal-01707294

\author{
HAL Id: hal-01707294 \\ https://hal.inria.fr/hal-01707294
}

Submitted on 12 Feb 2018

HAL is a multi-disciplinary open access archive for the deposit and dissemination of scientific research documents, whether they are published or not. The documents may come from teaching and research institutions in France or abroad, or from public or private research centers.
L'archive ouverte pluridisciplinaire HAL, est destinée au dépôt et à la diffusion de documents scientifiques de niveau recherche, publiés ou non, émanant des établissements d'enseignement et de recherche français ou étrangers, des laboratoires publics ou privés. 


\title{
A Framework for Mathematical Analysis of Collaborative SCM in ColPMan Game
}

\author{
Tatsuki Furukawa, Tomomi Nonaka, and Hajime Mizuyama \\ Management Technology Course, Aoyama Gakuin University, \\ 5-10-1 Fuchinobe, Chuo-Ku, Sagamihara, Kanagawa 252-5258, Japan \\ ifuruka.16.5@gmail.com, \{nonaka, mizuyama $\}$ @ise.aoyama.ac.jp
}

\begin{abstract}
ColPMan is a multi-player serious game through which a team of players can experientially learn how to collaboratively operate a virtual in-house supply chain. In this game, the problem of operating the whole chain is divided into sub-problems and each of them is addressed by a different player. While playing the game, the sub-problems distributed to the players can be linked to one another in a certain way through communication among them. This paper provides a framework for mathematically analyzing the effects of (the way the sub-problems are linked through) the communication. This also clarifies what the players should discuss and learn in the debriefing session.
\end{abstract}

Keywords: experiential learning, collaboration, negotiation, SCM, serious game

\section{Introduction}

Serious games have been successfully used for experiential learning and training in various fields including supply chain management. One of the most wellknown examples of such a game in SCM is Beer Game (Sterman 1989), through which the bullwhip effect can be taught. It treats a supply chain as a network of stock points, and deals only with ordering decisions. However, in a large-scale in-house supply chain, the managers of different sites of the chain need to make not only ordering decisions but also production and delivery schedules, which are interrelated to one another. How to collaboratively make such decisions in an uncertain market environment is a big challenge. Thus, the authors have proposed a multi-player serious game named ColPMan, in which a team of players can experientially learn how to collaboratively operate a large-scale in-house supply chain (Furukawa et al. 2016, Mizuyama et al. 2016, Nonaka et al. 2016).

In a mathematical sense, operational decisions to be made for running a supply chain can be captured as a sort of combinatorial optimization problem. Since it is usually too huge to be addressed by a single decision maker, the whole problem is divided into sub-problems of manageable sizes and they are handled by different sites of the chain. However, it is often inefficient in practice to simply deal with the sub-problems individually without coordinating the interdependence among them adaptively in an uncertain environment. This is why collaboration among the managers of the sites is desirable and important. However, 


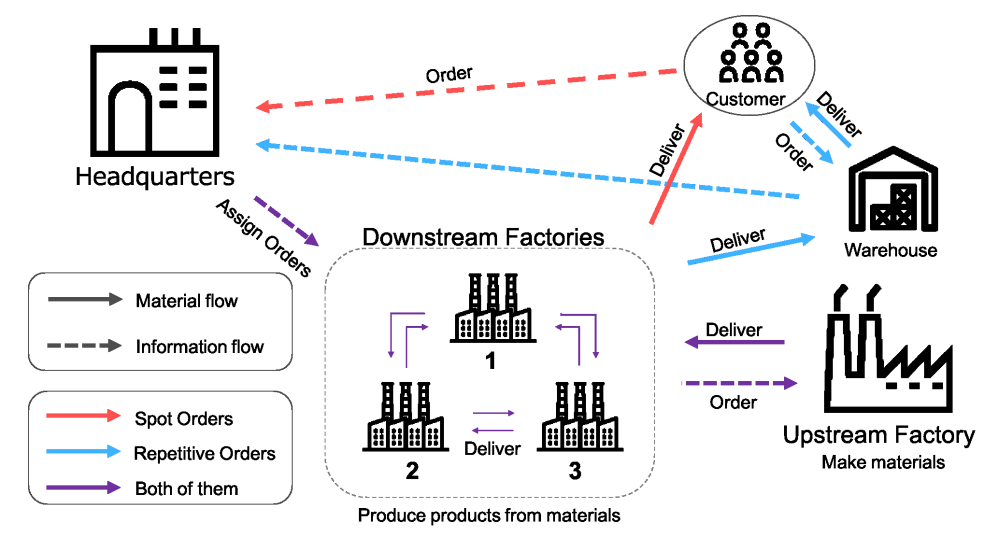

Fig. 1. Supply Chain Model

the desirable collaboration remains to be a vague concept, and should be refined into a well-defined one so that the ColPMan players can learn. Thus, this paper deems the collaboration as linking the sub-problems adaptively through communication, and provides a framework for mathematically analyzing the effects of the way the sub-problems are linked through the communication in ColPMan game.

\section{Outline of ColPMan Game}

There are two types of orders, repetitive and spot. The supply chain is composed of five main sites; a headquarters (HQ) which accepts orders from customers, three downstream factories (DSFs) which make products, and an upstream factory (USF) which manufactures materials for the products, as shown in Fig. 1. HQ assigns spot orders received from customers and repetitive orders to fill inventory to DSFs. Each DSF makes assigned products from materials and places material orders to USF. USF creates and delivers the materials to DSFs. After a product is made, it is delivered to the customer if it is a spot-ordered, and is kept in the warehouse until being pulled by the customer if it is repetitiveordered. Each DSF and USF can hold the materials as inventory until processed or delivered.

Each player is assigned to one of the sites and makes its operational decisions such as production schedules. The time axis is divided into terms and periods, where one term consists of five periods. At the beginning of each term, HQ player assigns orders to DSFs, and DSF players make the production schedules for delivering ordered products to customers by their due dates and order some materials. USF player determines the material manufacturing schedule and material delivery plan. After they finished inputting those decisions, how the supply chain operations progress according to those decisions under uncertainty is calculated by a computer simulation. The uncertainty includes arrivals of new spot orders, variations in processing times, and occurrence of defectives. This cycle 
Table 1. Notations for HQ's Sub-Problem

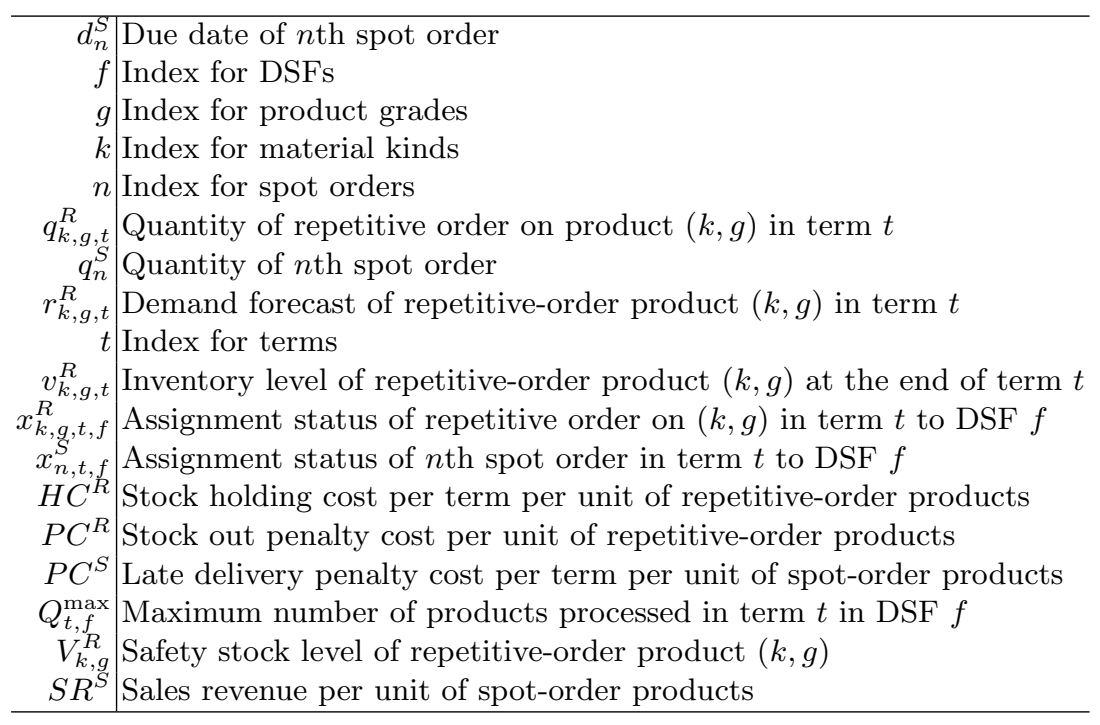

is repeated for a preset number of terms. At the beginning of each period, each player observes the operational progress and can modify the corresponding plans if necessary. The game score is defined by the sales revenue of fulfilled spot orders minus inventory holding costs, setup costs, late delivery penalty costs, and stock out penalty costs.

\section{Formulation of Individual Subproblems}

\subsection{Order Planning Problem Addressed by HQ}

HQ is not directly given the detailed information on the structure of manufacturing costs and production schedules in factories, and is expected to assign orders to DSFs so as to maximize the revenue. Thus, in the baseline problem addressed by HQ, order assignments $x_{k, g, t, f}^{R}$ and $x_{n, t, f}^{S}$ are determined so that the objective function

$$
\begin{aligned}
& \sum_{n} q_{n}^{S} \sum_{t}\left[S R^{S} \sum_{f} x_{n, t, f}^{S}-\max \left(0, t-d_{n}^{S}\right) P C^{S} \sum_{f} x_{n, t, f}^{S}\right] \\
& \quad-\sum_{k} \sum_{g} \sum_{t} q_{k, g, t}^{R}\left[P C^{R}\left(1-\sum_{f} x_{k, g, t, f}^{R}\right)+\frac{H C^{R}}{2} \sum_{f} x_{k, g, t, f}^{R}\right]
\end{aligned}
$$


Table 2. Notations for DSFs' Sub-Problem

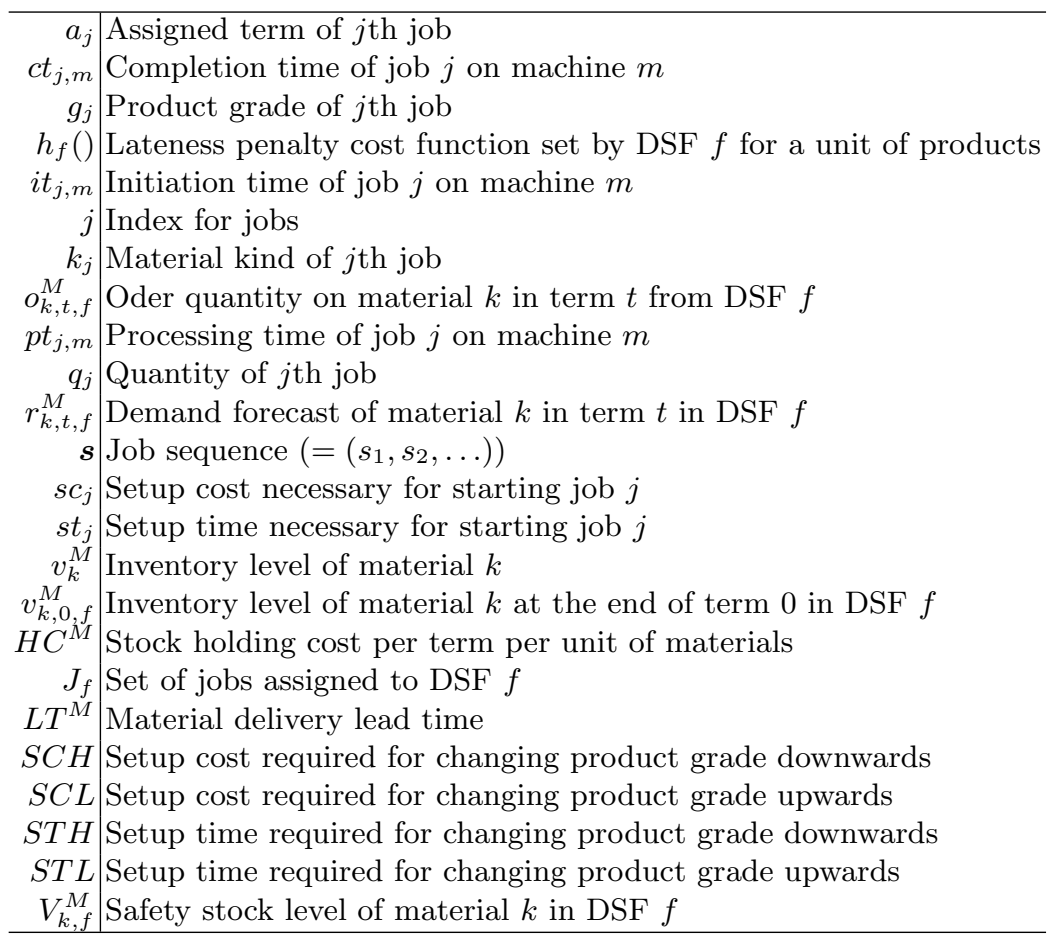

should be maximized under the following constraints.

$$
\begin{gathered}
\sum_{f} x_{k, g, t, f}^{R} \leq 1 \quad \forall(k, g, t) \\
\sum_{t} \sum_{f} x_{n, t, f}^{S} \leq 1 \quad \forall n \\
\sum_{n} q_{n}^{S} \cdot x_{n, t, f}^{S}+\sum_{k} \sum_{g} q_{k, g, t}^{R} \cdot x_{k, g, t, f}^{R} \leq Q_{t, f}^{\max } \quad \forall(t, f)
\end{gathered}
$$

It is assumed that $q_{k, g, t}^{R}$ is approximated by

$$
\begin{aligned}
& q_{k, g, 1}^{R}=r_{k, g, t}^{R}+V_{k, g}^{R}-v_{k, g, 0}^{R} \\
& q_{k, g, t}^{R}=r_{k, g, t}^{R} \quad(t=2,3)
\end{aligned}
$$

\subsection{Scheduling and Order Planning Problems Addressed by DSFs}

Each DSF needs to determine its production schedule and material order plan so as to minimize related costs. Since the actual lateness penalty costs caused for each job is not directly visible to the DSF (but only to HQ), an arbitrary function is used here. The baseline problem addressed by each DSF $f$ is a two 
machine flow shop scheduling problem, where the jobs are the spot and repetitive orders assigned to DSF $f$ by HQ. In this problem, the objective function to be minimized is

$$
\begin{aligned}
& \sum_{j \in J_{f}} s c_{j}+H C^{M}\left[\sum_{j \in J_{f}} q_{j} \cdot i t_{j, 1}+\sum_{k} \sum_{t} o_{k, t, f}^{M} \cdot(4-t)\right] \\
& \quad+\sum_{j \in J_{f}} q_{j} \cdot h_{f}\left(c t_{j, 2}-a_{j}\right)
\end{aligned}
$$

and the decision variable is the job sequence $\boldsymbol{s}$. When a job sequence $\boldsymbol{s}$ is given, setup costs $s c_{j}$ and times $s t_{j}$ are determined as

$$
\left(s c_{s_{i}}, s t_{s_{i}}\right)= \begin{cases}(S C L, S T L) & \left(g_{s_{i-1}}>g_{s_{i}}\right) \\ (0,0) & \left(g_{s_{i-1}}=g_{s_{i}}\right) \\ (S C H, S T H) & \left(g_{s_{i-1}}<g_{s_{i}}\right)\end{cases}
$$

where $s_{0}$ is the last job processed in term 0 . Further, the corresponding initiation times $i t_{j, m}$, completion times $c t_{j, m}$, and material order quantities $o_{k, t, f}^{M}$ can be calculated as follows.

Step 0: Set $v_{k}^{M}=v_{k, 0, f}^{M}, o_{k, 1, f}^{M}=o_{k, 2, f}^{M}=o_{k, 3, f}^{M}=0 \forall k, i=1, t=1$.

Step 1: Calculate the initiation and completion times of job $s_{i}$ as

$$
\begin{aligned}
i t_{s_{i}, 1} & = \begin{cases}L T^{M} & \left(c t_{s_{i-1}, 1}<L T^{M} \wedge v_{k_{s_{i}}}^{M}<q_{s_{i}}\right) \\
\max \left(0, c t_{s_{i-1}, 1}\right) & (\text { otherwise })\end{cases} \\
c t_{s_{i}, 1} & =i t_{s_{i}, 1}+p t_{s_{i}, 1} \\
i t_{s_{i}, 2} & =\max \left(c t_{s_{i}, 1}, c t_{s_{i-1}, 2}+s t_{s_{i}}\right) \\
c t_{s_{i}, 2} & =i t_{s_{i}, 2}+p t_{s_{i}, 2}
\end{aligned}
$$

and update the inventory level as $v_{k_{s_{i}}}^{M}=v_{k_{s_{i}}}^{M}-q_{s_{i}}$. Further, if $c t_{s_{i}, 1} \geq t+L T^{M}$ holds, then set $o_{k, t, f}^{M}=\max \left(0, V_{k, f}^{M}-v_{k}^{M}\right) \forall k, v_{k}^{M}=\max \left(v_{k}^{M}, V_{k, f}^{M}\right) \forall k$, and $t=t+1$.

Step 2: If $i=\left|J_{f}\right|$ go to Step 3. Otherwise, go back to Step 1 after setting $i=i+1$.

Step 3: If $t \leq 3$, set $o_{k, 3, f}^{M}=\max \left(0, V_{k, f}^{M}-v_{k}^{M}+r_{k, 4, f}^{M} \cdot L T^{M}\right)$.

\subsection{Scheduling and Delivery Planning Problems Addressed by USF}

USF needs to determine its production schedule and material delivery plan so as to minimize related costs, where an arbitrary value is set and used as the shortage penalty cost per unit of materials. The baseline problem addressed by USF is a sort of single machine scheduling problem. In this problem, the objective 
Table 3. Notations for USF's Sub-Problem

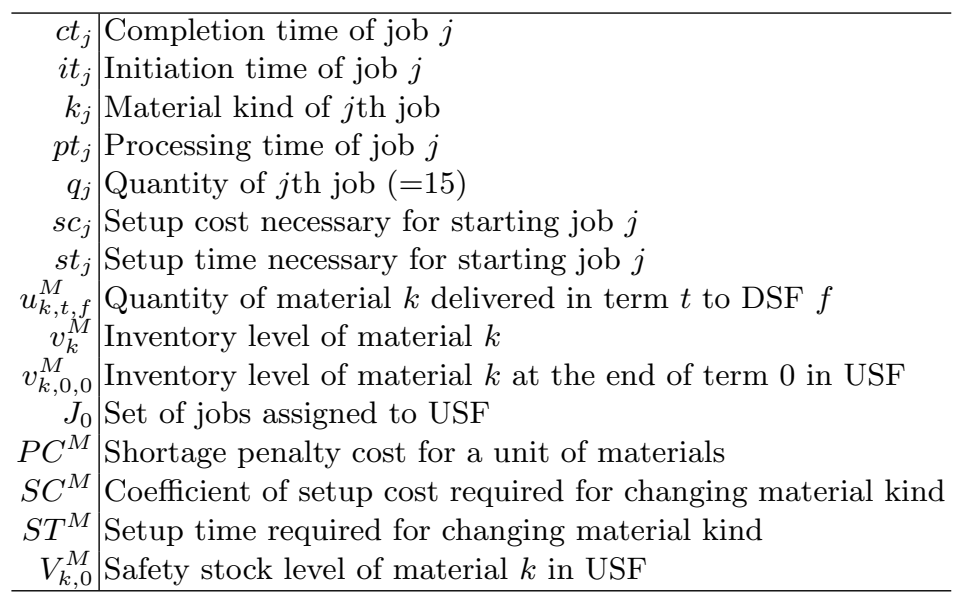

function to be minimized is

$$
\begin{aligned}
& \sum_{j \in J_{0}} s c_{j}+H C^{M}\left[\sum_{j \in J_{0}} q_{j} \cdot\left(3-c t_{j}\right)-\sum_{k} \sum_{t} \sum_{f} u_{k, t, f}^{M} \cdot(4-t)\right] \\
& \quad+P C^{M} \sum_{k} \sum_{t} \sum_{f}\left(o_{k, t, f}^{M}-u_{k, t, f}^{M}\right)
\end{aligned}
$$

and the decision variable is the job sequence $s$. When a job sequence $s$ is given, setup costs $s c_{j}$ are determined as

$$
s c_{s_{i}}=S C^{M} \cdot\left|k_{s_{i}}-k_{s_{i-1}}\right|
$$

where $s_{0}$ is the last job processed in term 0 . Further, the corresponding initiation times $i t_{j}$, completion times $c t_{j}$, material delivery quantities $u_{k, t, f}^{M}$ can be calculated as follows.

Step 0: Set $v_{k}^{M}=v_{k, 0,0}^{M}, i=0, t=0$.

Step 1: If $\max \left(0, c t_{s_{i}}\right) \geq t$ holds, go to Step 2. Otherwise, go to Step 3.

Step 2: If $t=3$ holds, finish the procedure. Otherwise, set $t=t+1$ and determine $u_{k, t, f}^{M}$ by minimizing

$$
P C^{M} \sum_{k} \sum_{f}\left(o_{k, t, f}^{M}-u_{k, t, f}^{M}\right)
$$

subject to

$$
\begin{aligned}
\sum_{f} u_{k, t, f}^{M} & \leq v_{k}^{M} & (\forall k) \\
u_{k, t, f}^{M} & \leq o_{k, t, f}^{M} & \forall(k, t, f)
\end{aligned}
$$


and update the inventory levels as

$$
v_{k}^{M}=v_{k}^{M}-\sum_{f} u_{k, t, f}^{M}
$$

Step 3: Update the inventory level as $v_{k_{s_{i}}}^{M}=v_{k_{s_{i}}}^{M}+q_{s_{i}}$ and set $i=i+1$. Calculate the initiation and completion times of job $s_{i}$ as

$$
\begin{aligned}
i t_{s_{i}} & =\max \left(0, c t_{s_{i-1}}\right)+s t_{s_{i}} \\
c t_{s_{i}} & =i t_{s_{i}}+p t_{s_{i}}
\end{aligned}
$$

Go back to Step 1.

\section{Possible Linkages among Sub-problems}

\subsection{Sharing Inventory Information}

There are some parameters, which can be exchanged by the communication among the sites and change the form of each sub-problem. We call them as link-parameters and sort them out based, for example, on the communication logs obtained from the game sessions conducted in the past. One of the most frequently mentioned information was on material inventory. Possible linkage patterns based on material inventory information can be classified as follows.

Pattern 1: DSFs share the material inventory levels with HQ

Pattern 2: USF shares the material inventory levels with HQ

Pattern 3: USF shares the material inventory levels with DSFs

The inventory levels shared here may include not only the current levels but also the future levels estimated with ordering, production and delivery plans.

In pattern 1 and 2, the inventory levels shared with HQ will pose additional constraints on the quantity of products of each material type assignable to each DSF. It is also possible to add a term corresponding to the material inventory costs to the objective function and thereby enhance the turnover rate of the materials. In pattern 3, equation (9) can be modified incorporating the shared information.

\subsection{Sharing Demand Information}

Demand related information is also appeared often in the communication log. Possible linkage pattens based on demand information can be classified as follows.

Pattern 4: HQ shares the future demand with DSFs

Pattern 5: HQ shares the future demand with USF

Pattern 6: DSFs share the future demand with USF

Information sharing of pattern 4 and 6 is formally made between the corresponding sites for three terms. However, it will make a difference whether the future demand values informed are fixed or not. USF can utilize the information shared in pattern 5 for double check. 


\subsection{Sharing Other Information}

If $\mathrm{HQ}$ is notified that a setup operation is required when changing product grades in DSFs and when changing material kinds in USF, HQ will try to reduce the number of setup operations necessary by adding appropriate penalty terms to its objective function (1). Further, if it is informed that the processing times $p t_{j, 2}$ are dependent not only on the grades but also on the DSFs, HQ can refine the constraint (4) by incorporating the difference. The lateness penalty cost function $h_{f}()$ set in each DSF and the shortage penalty cost per unit $P C^{M}$ set in USF can be refined if the factory obtains information on how the actual cost changes according to the completion time of the job from HQ.

\section{Conclusions}

The problem of operating the whole supply chain is divided into sub-problems, and they are handled by different players in ColPMan game. While playing the game, the sub-problems may be linked to one another in a certain way through communication among the players. How they are linked together affects the performance but how they should be has not been clearly understood. Thus, this paper first mathematically formulates each sub-problem, and provides a framework for analyzing the effects of the way the sub-problems are linked. Next step will be to compare the performance of the chain under different linkage types through numerical experiments. Since the relative performance may be dependent on the environmental conditions, the numerical comparison should be made in different conditions.

\section{References}

Furukawa, T., Nonaka, T., and Mizuyama, H.: A GWAP Approach to Analyzing Informal Algorithm for Collaborative Group Problem Solving, The Fourth AAAI Conference on Human Computation and Crowdsourcing: HCOMP 2016, Austin, USA, Nov. (2016)

Mizuyama, H., Nonaka, T., Yoshikawa Y., and K. Miki.: ColPMan: A Serious Game for Practicing Collaborative Production Management, Simulation and Gaming in the Network Society, Edited by T. Kaneda, H. Kanegae, Y. Toyoda and P. Rizzi, Springer, pp.185-197 (2016)

Nonaka, T., Miki, K., Odajima, R., and Mizuyama, H.: Analysis of Dynamic Decision Making Underpinning Supply Chain Resilience: A Serious Game Approach, Proceedings of the 13rd IFAC/IFIP/IFORS/IEA Symposium on Analysis, Design, and Evaluation of Human-Machine Systems, Kyoto Japan, Sep. (2016)

Sterman, J.D.: Modeling Managerial Behavior: Misperceptions of Feedback in a Dynamic Decision Making Experiment, Management Science, Vol.35, pp.321-339 (1989) 